ARTICLE

https://doi.org/10.1038/s41467-019-10985-5

\title{
Atomic-level passivation mechanism of ammonium salts enabling highly efficient perovskite solar cells
}

\author{
Essa A. Alharbi ${ }^{1}$, Ahmed Y. Alyamani ${ }^{2}$, Dominik J. Kubicki ${ }^{1,3}$, Alexander R. Uh1 1,6, Brennan J. Walder ${ }^{3}$, \\ Anwar Q. Alanazi ${ }^{1}$, Jingshan Luo (1) 1,7, Andrés Burgos-Caminal ${ }^{4}$, Abdulrahman Albadri², Hamad Albrithen ${ }^{2,5}$,

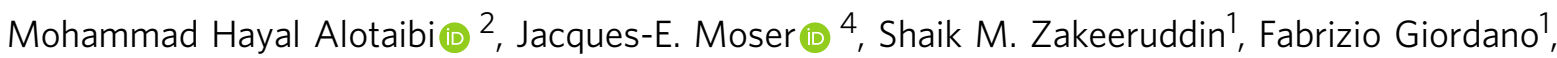 \\ Lyndon Emsley ${ }^{3} \&$ Michael Grätzel ${ }^{1}$
}

The high conversion efficiency has made metal halide perovskite solar cells a real breakthrough in thin film photovoltaic technology in recent years. Here, we introduce a straightforward strategy to reduce the level of electronic defects present at the interface between the perovskite film and the hole transport layer by treating the perovskite surface with different types of ammonium salts, namely ethylammonium, imidazolium and guanidinium iodide. We use a triple cation perovskite formulation containing primarily formamidinium and small amounts of cesium and methylammonium. We find that this treatment boosts the power conversion efficiency from $20.5 \%$ for the control to $22.3 \%, 22.1 \%$, and $21.0 \%$ for the devices treated with ethylammonium, imidazolium and guanidinium iodide, respectively. Best performing devices showed a loss in efficiency of only $5 \%$ under full sunlight intensity with maximum power tracking for $550 \mathrm{~h}$. We apply 2D- solid-state NMR to unravel the atomiclevel mechanism of this passivation effect.

\footnotetext{
${ }^{1}$ Laboratory of Photonics and Interfaces, Institute of Chemical Sciences and Engineering, School of Basic Sciences, Ecole Polytechnique Fédérale de Lausanne, CH-1015 Lausanne, Switzerland. ${ }^{2}$ National Center for Nanotechnology, King Abdulaziz City for Science and Technology, Riyadh 11442, Saudi Arabia.

${ }^{3}$ Laboratory of Magnetic Resonance, Institute of Chemical Sciences and Engineering, School of Basic Sciences, Ecole Polytechnique Fédérale de Lausanne, CH-1015 Lausanne, Switzerland. ${ }^{4}$ Photochemical Dynamics Group, Institute of Chemical Sciences and Engineering, Lausanne Centre for Ultrafast Science, École polytechnique fédérale de Lausanne, CH-1015 Lausanne, Switzerland. ${ }^{5}$ Physics and Astronomy Department-Research Chair for Tribology, Surface and Interface Sciences, College of Science, and King Abdullah Institute for Nanotechnology-Aramco Laboratory for Applied Sensing Research, King Saud University, Riyadh 11451, Saudi Arabia. ${ }^{6}$ Present address: Laboratory for Solar Energy and Fuels, The University of British Columbia, Kelowna, BC V1V 1V7, Canada. ${ }^{7}$ Present address: Institute of Photoelectronic Thin Film Devices and Technology, Key Laboratory of Photoelectronic Thin Film Devices and Technology of Tianjin, Nankai University, 300350 Tianjin, China. Correspondence and requests for materials should be addressed to F.G. (email: fabrizio. giordano@epfl.ch) or to L.E. (email: Iyndon.emsley@epfl.ch) or to M.G. (email: michael.graetzel@epfl.ch)
} 
M etal halides perovskite are one of the most promising light harvesting materials among emerging photovoltaic technologies ${ }^{1-5}$ solar to electric power conversion efficiencies (PCEs) reaching presently $23.7 \%{ }^{6}$. Their ease of manufacturing together with low cost fabrication and high performance have made metal halide perovskites a true breakthrough in the thin film solar cell technology. However, solution deposition methods are prone to produce pinholes and defects whether at the grain boundaries or at the surface, which is considered one of the reasons behind a low device performance and stability ${ }^{7,8}$. One major obstacle to the development and commercialization of this technology continues to be the operational stability of the photovoltaic devices. Despite the vast progress that has been achieved on the synthesis of high quality multi-crystalline films, some complex problems have only been partially mitigated ${ }^{3}$. The often observed hysteretic behavior during $J-V$ characterization, caused by ion mobility, can be considered a primary indication for the intrinsic long-term steady-state instability 9 . In that respect, the phase stabilization of specific perovskite formulations has been the object of thorough investigation ${ }^{2,10,11}$. Recently, the reduction of defects at the surface and grain boundaries of the perovskite film as well as at the interfaces with the electrical contacts, has attracted great interest for its dramatic impact on the operational stability and efficiency of the device 7,12 . Mitigation of surface defects, whether at the interface of perovskite/HTL or perovskite/ electron transport layer (ETL), provides the added benefit of improving the open-circuit voltage $\left(V_{\text {oc }}\right)$ without affecting the charge carrier transport or the fill factor (FF). Previous studies have employed several types of ammonium cations in order to impede the charge carrier recombination losses occurring at the interfaces or throughout the bulk of the perovskite film. Among those, formamidinium bromide ( $\mathrm{FABr}$ ) was used as an electron blocking layer, forming a wide band gap over layer $\left(\mathrm{FAPbBr}_{3-\mathrm{x}} \mathrm{I}_{\mathrm{x}}\right)$ at the interface between the perovskite/HTL and consequently improving $V_{\mathrm{oc}}$ by approximately $60 \mathrm{mV}^{13}$. In a similar manner methylammonium iodide (MAI) was thermally evaporated at the interface perovskite/HTL, enhancing efficiency from $14.5 \%$ to $17.2 \%$ with a high reproducibility ${ }^{14}$. Lately, quaternary ammonium halides were found to decrease the ionic defects at the perovskite surface and significantly improve efficiency and stability $^{7}$. Furthermore, phenylalkylamine molecules ${ }^{15,16}$, and polymers ${ }^{17}$ have been used to improve the efficiency and moisture tolerance of perovskite solar cells (PSC). Moreover, guanidinium $^{18-21}$, ethylammonium ${ }^{22,23}$, and imidazolium ${ }^{24,25}$ have been used as additive to improve different aspects of PSC operation.

Herein we report a facile strategy to tailor the interface between the perovskite and the HTL (Fig. 1). We show that the modification of the perovskite surface via the addition of organic ammonium salts, namely, ethylammonium iodide $\left(\left[\left(\mathrm{C}_{2} \mathrm{H}_{5}\right) \mathrm{NH}_{3}\right]\right.$ I, (EAI)), imidazolium iodide $\left(\left[\mathrm{C}_{3} \mathrm{~N}_{2} \mathrm{H}_{5}\right] \mathrm{I}\right.$, (IAI)), and guanidinium iodide $\left(\left[\mathrm{C}\left(\mathrm{NH}_{2}\right)_{3}\right] \mathrm{I},(\mathrm{GuaI})\right)$, considerably increases the device performance. We use mixed-cation/halide perovskite formulations of the composition $\left(\mathrm{FA}_{0.9} \mathrm{Cs}_{0 \cdot 07} \mathrm{MA}_{0 \cdot 03} \mathrm{~Pb}\right.$ $\left.\left(\mathrm{I}_{0.92} \mathrm{Br}_{0.08}\right)_{3}\right)$ with $3 \%$ excess of $\mathrm{PbI}_{2}$. These agents greatly reduce the hysteresis in the $J V$ curve improving the solar to electric power conversion efficiency from $20.5 \%$ for the control device to $22.3,22.1$, and $21.0 \%$ for the EAI-, IAI-, and GuaI-treated device, respectively. Moreover, defect mitigation improves the operational stability for the PSC's, which was tested at full solar intensity under maximum power tracking condition for $550 \mathrm{~h}$ with a small loss of only $5 \%$ for the best performing devices.

\section{Results}

Characterization and fabrication of perovskite thin films. We employed a device architecture comprising an FTO glass substrate, on top of which we deposited a compact $\mathrm{TiO}_{2}$ layer followed by mesoporous $\mathrm{TiO}_{2} /$ perovskite/passivation layer/spiroOMeTAD/gold. The complete procedure for the device fabrication is detailed in the method section. In short, the perovskite film was annealed at $150^{\circ} \mathrm{C}$ for $30-40 \mathrm{~min}$. After cooling down to room temperature, the passivation layer was immediately deposited by spin-coating a solution of ammonium salt (EAI, IAI, and GuaI) in isopropanol and subsequent annealing at $70^{\circ} \mathrm{C}$ for 10-15 min. The concentration of the solution was optimized for each compound investigated (Supplementary Table 1) and the different treatments were compared at their best condition. We note that the high annealing temperature could potentially drive off methylammonium from the thin film. Solid-state NMR quantification ${ }^{26}$ of the cation content of our films yielded 0.97 mol \% FA and $0.03 \mathrm{~mol} \% \mathrm{MA}$, which agrees with the stoichiometry of the precursor solution, indicating that MA is fully retained in the final perovskite composition even after the high annealing (Supplementary Fig. 1 and Supplementary Note 1).

The surface treatment described above significantly modifies the perovskite composition and surface morphology due to a chemical reaction with the perovskite. This was traced by crystallographic structure analysis of the perovskite film using X-ray diffraction (XRD). Fig. 2a and Supplementary Fig. 2a, b, c, reveal the presence of unreacted $\mathrm{PbI}_{2}\left(2 \theta=12.7^{\circ}\right)$ which is expected, since the precursor solution contains $3 \%$ excess of $\mathrm{PbI}_{2}$. All three surface treatments have a similar effect on the XRD pattern. In particular, for concentrations above $3 \mathrm{mg} / \mathrm{ml}$ for all treatments, the $\mathrm{PbI}_{2}$ peaks completely disappear (Fig. 2a and Supplementary Fig. $2 a-c)$, presumably via formation of a thin non-perovskite cover layer of EA/IA/Gua lead halide on top of the perovskite film. We have recently shown that solid-state magic angle spinning (MAS) NMR can be used to probe this kind of atomic-level microstructure of multi-component lead halide perovskites $^{21,26-28}$. We therefore test the hypothesis by carrying out solid-state NMR measurements on a thin film of $\mathrm{FA}_{0.93} \mathrm{Cs}_{0 \cdot 07} \mathrm{PbI}_{3}$ (further referred to as $\mathrm{CsFA}(\mathrm{I})$ ) treated with EAI $\left(5 \mathrm{mg} \mathrm{ml}^{-1}\right)$. We used the pure-iodide CsFA(I) composition to avoid the overlap between MA and EA ${ }^{1} \mathrm{H}$ signals and the

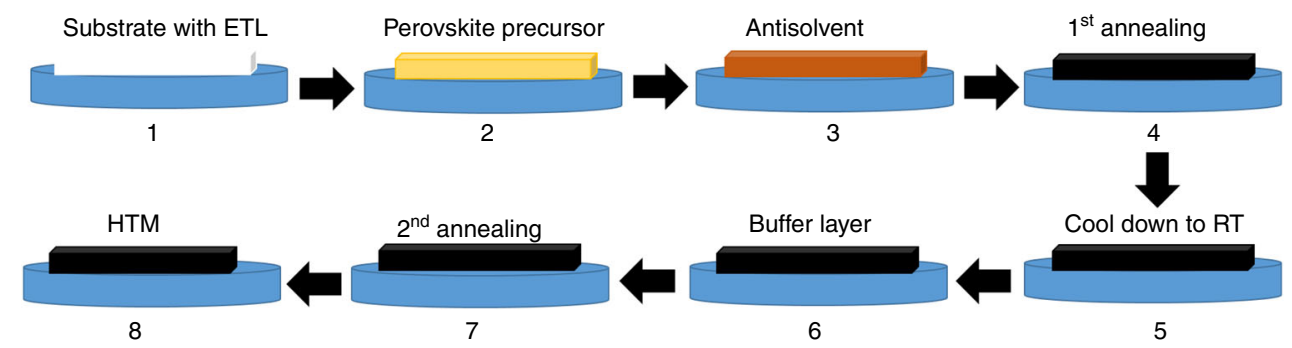

Fig. 1 Scheme of our method for treating perovskite films by spin-coating of different organic ammonium salts (EAI, IAI, and Gual) and consecutive annealing 
a

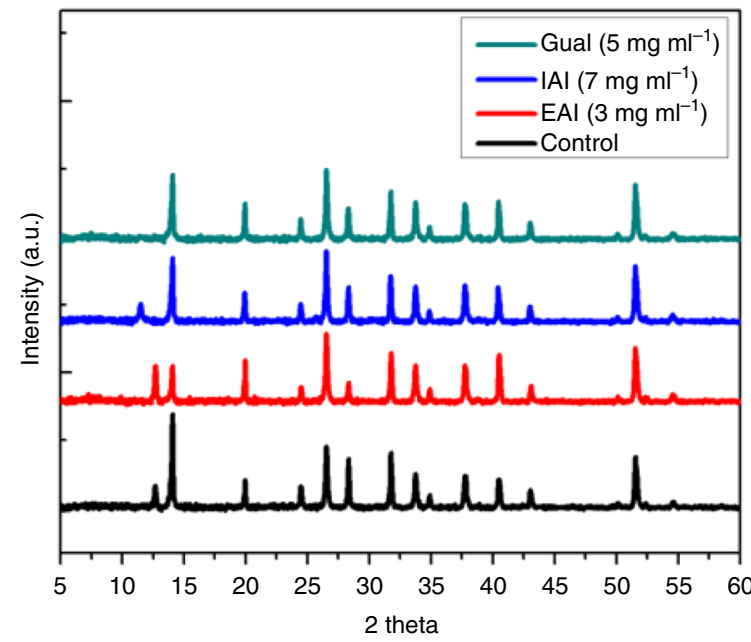

g

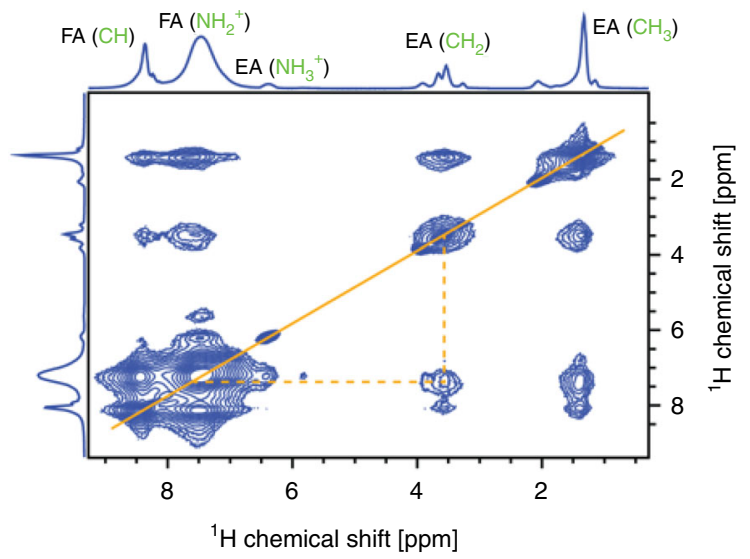

b

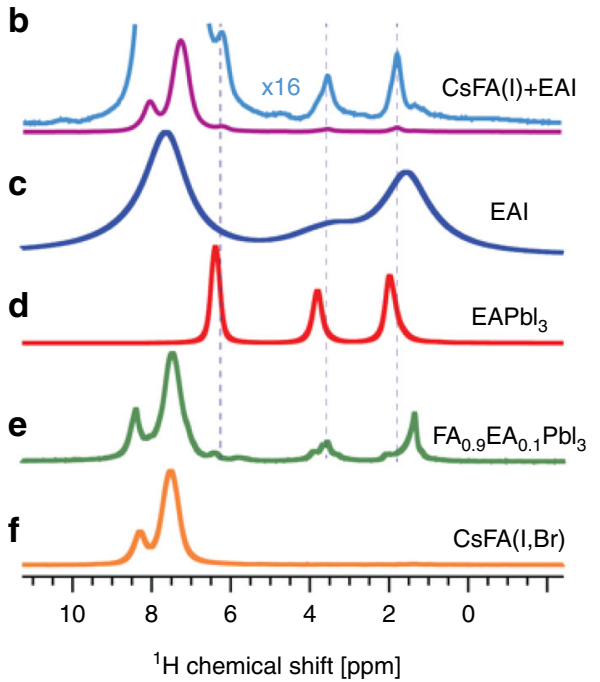

h

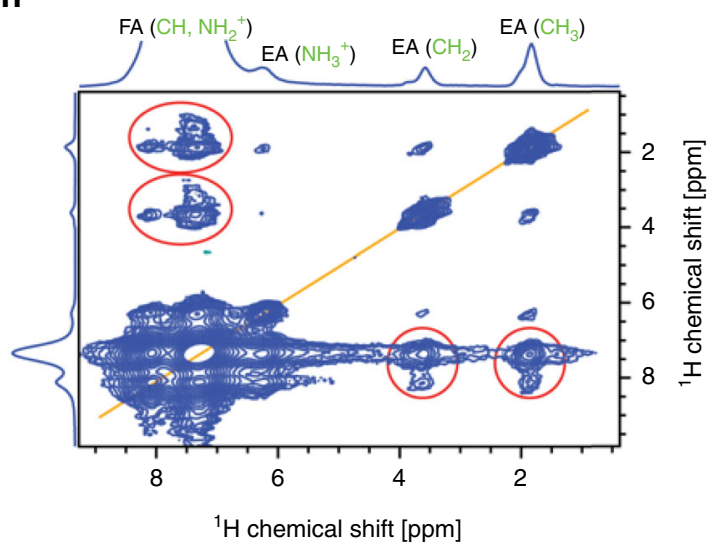

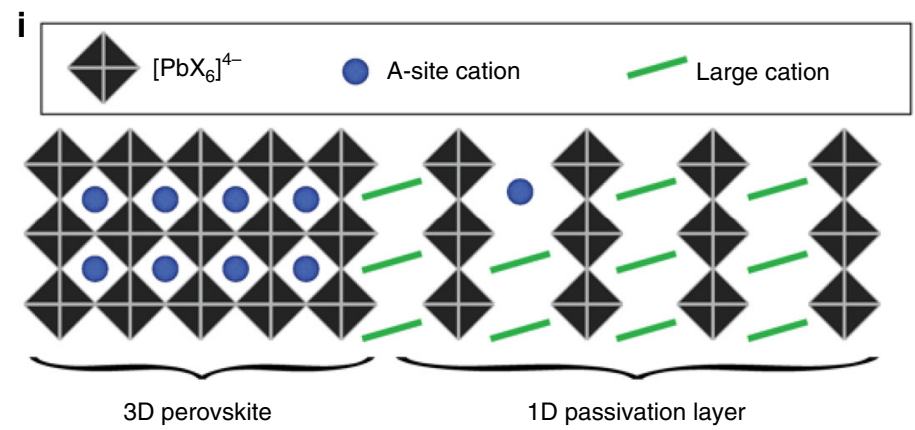

Fig. 2 Structural characterization of the passivated perovskite films: a XRD patterns of the treated films compared to the control. Solid-state ${ }^{1} \mathrm{H} M A S$ NMR measurements at $21.1 \mathrm{~T}, 300 \mathrm{~K}$ and $20 \mathrm{kHz}$ MAS (unless noted otherwise): $\mathbf{b}$ thin film of CsFA(I) treated with $5 \mathrm{mg} \mathrm{ml}^{-1} \mathrm{EAI}$ (at $60 \mathrm{kHz} \mathrm{MAS),} \mathbf{c}$ neat EAl,

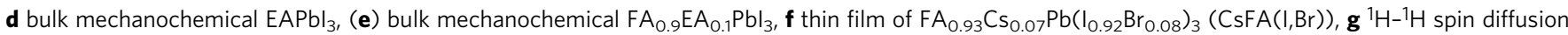
(20 kHz MAS) experiment evidencing atomic-level proximity between FA and EA in the mixed FA/EA phase; one of the EA/FA cross-peaks has been indicated with dashed lines. $\mathbf{h}^{1} \mathrm{H}-{ }^{1} \mathrm{H}$ spin diffusion experiment (60 kHz MAS) evidencing atomic-level proximity between $F A$ and EA in the $\mathrm{FA}_{0.93} \mathrm{Cs}_{0.07} \mathrm{Pbl}_{3}$, thin film treated with $5 \mathrm{mg} \mathrm{ml}^{-1} \mathrm{EAl}$ (FA/EA cross-peaks in red circles), $\mathbf{i}$ schematic representation of the 1D/3D heterostructure evidenced by solid-state NMR proximity measurements

formation of mixed iodide-bromide phases, which would complicate the interpretation. Figure $2 \mathrm{~b}$ shows a ${ }^{1} \mathrm{H}$ solid-state MAS NMR spectrum of the CsFA(I) thin film treated with $5 \mathrm{mg} \mathrm{ml}^{-1}$ EAI and identifies the presence of two organic cations in the film: FA (8.1 ppm $(\mathrm{CH})$ and $7.3 \mathrm{ppm}\left(\mathrm{NH}_{2}{ }^{+}\right)$) as well as EA (6.3 ppm $\left(\mathrm{NH}_{3}{ }^{+}\right), 3.6 \mathrm{ppm}\left(\mathrm{CH}_{2}\right)$ and $\left.1.8\left(\mathrm{CH}_{3}\right)\right)$. There is no unreacted EAI in the film (Fig. 2c), which would give signals at 7.7, 3.3, and $1.6 \mathrm{ppm}$ with a full width at half maximum (FWHM) of 1.5-2.0 ppm due to strong ${ }^{1} \mathrm{H}-{ }^{1} \mathrm{H}$ dipole-dipole couplings. The EA signals in the thin film have FWHM of $0.1-0.2 \mathrm{ppm}$, consistent with lower ${ }^{1} \mathrm{H}$ density in the EA-containing phase ${ }^{29}$. The new EA species in the thin film have a spectral signature similar to that of $\mathrm{EAPbI}_{3}$ (Fig. 2d, 6.4, 3.8, and $2.0 \mathrm{ppm}$ ). We thus confirm that EAI is fully converted into EA-containing lead halide phases during the passivation treatment.

That said, the EA signals are slightly shifted, indicating a small structural difference with respect to the pure $1 \mathrm{D} \mathrm{EAPbI}_{3}$ phase $^{29}$. This can be potentially caused by the formation of mixed FA/EA, 
Cs/EA, and/or Cs/EA/FA phases. We demonstrate this by preparing $\alpha-\mathrm{FAPbI}_{3}$ substitutionally doped with $10 \mathrm{~mol} \%$ EAI $\left(\mathrm{FA}_{0.9} \mathrm{EA}_{0.1} \mathrm{PbI}_{3}\right)$ (Fig. 2e). The spectrum of this material shows a distribution of environments for each of the EA sites $\left(\mathrm{CH}_{3}\right.$ : 0.6-2.3 ppm, $\mathrm{CH}_{2}: 3.0-4.3$ and $\mathrm{NH}_{3}{ }^{+}:$5.3-6.7 ppm). This distribution is likely caused by the formation of mixed FA/EA structures with a varying number of FA slabs separated by EA spacers, in structures similar to those reported for 2-( $1 H$-pyrazol1 -yl)pyridine-doped $1 \mathrm{D} / 3 \mathrm{D}^{30}$ and butylammonium- and phenylethylammonium-based $2 \mathrm{D} / 3 \mathrm{D}$ materials ${ }^{31}$. We further evidence the formation of mixed FA/EA phases by performing two-dimensional ${ }^{1} \mathrm{H}-{ }^{1} \mathrm{H}$ spin diffusion experiments, which correlate signals based on their spatial proximity (up to around $10 \AA)^{32}$ (Fig. 2g). The peaks lying on the diagonal correspond to the species presented in the $1 \mathrm{D}$ projection above. On the other hand, off-diagonal peaks indicate that two chemical environments are in atomic-level proximity (an example is illustrated by the dashed orange line). Beside trivial intramolecular contacts, every EA environment $\left(\mathrm{CH}_{3}, \mathrm{CH}_{2}\right.$ and $\left.\mathrm{NH}_{2}\right)$ is correlated to each of the two FA environments $\left(\mathrm{CH}\right.$ and $\left.\mathrm{NH}_{2}{ }^{+}\right)$, demonstrating unambiguously that EA and FA are microscopically mixed within the same phase.

We then carried out the ${ }^{1} \mathrm{H}-{ }^{1} \mathrm{H}$ spin diffusion measurement on the EAI-treated CsFA(I) thin film and found through-space atomic-level contact between FA and EA (Fig. 2h, red circles), confirming that the formation of mixed EA/FA phases is general, regardless of the processing conditions. We also show that analogous atomic-level proximities are present in the case of IAI and GuaI treatment, confirming the formation of $3 \mathrm{D} / 1 \mathrm{D}$ heterostructures in these cases (Supplementary Figs. $7 \mathrm{~h}-\mathrm{m}$ and 8 ). While the exact spectral signature of EA in such mixed phases will depend on the FA/EA ratio and can conceivably be further modified by the presence of Cs, the chemical shift range and line widths corresponding to the model FA/EA phase matches that observed in the EAI-passivated thin film of CsFA(I). This finding is of paramount importance in that it shows that EAI is fully converted into new mixed EA/FA phases. Since the reaction is aided by IPA and as such happens without redissolution and recrystallization of the perovskite, the new EA/FA phase must form on the surface of the preexisting perovskite grains and its similarity from NMR to the $1 \mathrm{D} \mathrm{EAPbI}_{3}$ suggests it is a $1 \mathrm{D}$ structure. We note that it is not possible to quantify the EA/FA ratio in the $1 \mathrm{D}$ passivation layer by simply comparing it to reference $1 \mathrm{D} \mathrm{FA}_{1-\mathrm{x}} \mathrm{EA}_{\mathrm{x}} \mathrm{PbI}_{3}$ phases (Supplementary Fig. $7 \mathrm{c}-\mathrm{g}$ ), as in the $3 \mathrm{D} / 1 \mathrm{D}$ heterostructure the EA shift is additionally affected by the presence of the $3 \mathrm{D}$ perovskite phase in its immediate microscopic environment. This supports the formation of a firmly adhering passivation layer with high ambient stability, owing to the superior stability of $3 \mathrm{D}$ perovskites in hybrids with lower dimensionality structures (Fig. $2 \mathrm{i})^{31} \mathrm{We}$ also provide a comparison with a EA-treated film but without the second annealing step, which shows that the final 3D/1D heterostructure is formed during spin coating (Supplementary Fig. 7a-b). Finally, we note that solid-state ${ }^{1} \mathrm{H}$ MAS NMR quantification of the cation content in the passivated thin film revealed that the EA constitutes $10 \mathrm{~mol} \%$ of the total organic cation content (with $90 \mathrm{~mol} \% \mathrm{FA}$ ). After applying straightforward chemical and geometrical consideration, this corresponds to $27 \mathrm{~nm}$ thick $1 \mathrm{D}$ passivation layer (Supplementary Note 2).

Furthermore, surface morphology of the perovskite films was recorded via scanning electron microscopy (SEM) (Fig. 3a-d). In this study, the bulk perovskite composition was kept identical for all the conditions and only the surface was modified by the treatment with the 3 different agents. From Fig. $3 a-d$ and Supplementary Fig. 3 we infer that the surface of the treated films shows much smaller grains than the control sample. To rule out etching of the perovskite surface by IPA as a cause of the roughening, we examined a control film, which was treated by neat IPA and ascertained that the solvent on its own does not modify the perovskite surface morphology (Supplementary Fig. 3). We then investigate surface roughness of the perovskite films after the surface treatment using atomic force microscopy (AFM) (Fig. $3 \mathrm{f}-\mathrm{j}$ ). It is apparent that the surface roughness, and thereby the corresponding specific surface area, has increased significantly for all the treated samples, with GuaI $\left(5 \mathrm{mg} \mathrm{ml}^{-1}\right)$ showing the highest root mean square (RMS) roughness of $12.7 \mathrm{~nm}$, followed by EAI $\left(3 \mathrm{mg} \mathrm{ml}^{-1}\right)$ with $12.0 \mathrm{~nm}$, IAI $\left(7 \mathrm{mg} \mathrm{ml}^{-1}\right)$ with $9.00 \mathrm{~nm}$, and the control with $6.21 \mathrm{~nm}$.

Figure $4 \mathrm{a}$ and Supplementary Fig. 4 show that the absorbance onset for control/IPA corresponded to that of EAI, IAI, and GuaI-passivated films indicating that the band gap of the bulk perovskite was not noticeably affected by the surface treatment. We investigated the steady-state and time-resolved photoluminescence (PL) of the control and modified perovskite layer. Fig. $4 \mathrm{~b}$ and Supplementary Fig. 1a-d show an increase in PL intensity in response to the post treatments. This suggests a reduction of the non-radiative recombination losses that could be explained by defect mitigation induced by cation exchange and filling of iodide vacancies at the absorber surface in agreement with previous work on methylammonium lead iodide films ${ }^{14}$. It is worth mentioning that the GuaI when applied at $7 \mathrm{mg} \mathrm{ml}^{-1}$ and $10 \mathrm{mg} \mathrm{ml}^{-1}$ showed a slight red shift as shown in Supplementary Fig. 5.

We carried out time-resolved photoluminescence (TRPL) on glass $/ \mathrm{Al}_{2} \mathrm{O}_{3}$ /perovskite samples with and without surface treatment. We excited the sample from the from the perovskite side using $670 \mathrm{~nm}$ wavelength light. By fitting the luminescence decays in Fig. $4 c$ according to our previously reported procedure $\mathrm{e}^{27}$, we derived lifetimes $\tau_{1}=1 / k_{1}$ for the pseuo-first order trap mediated (Shockley-Read-Hall) non-radiative PL decay process. From TRPL data in Fig. 4c, the decay lifetime increased with the application of our surface treatment from $250 \mathrm{~ns}$ for the control to $560 \mathrm{~ns}, 625 \mathrm{~ns}$, and $333 \mathrm{~ns}$ for EAI $\left(3 \mathrm{mg} \mathrm{ml}^{-1}\right)$, IAI $\left(7 \mathrm{mg} \mathrm{ml}^{-1}\right)$, and GuaI ( $\left.5 \mathrm{mg} \mathrm{ml}^{-1}\right)$, respectively.

Photovoltaic device and performance. We examined the effect of surface passivation by ammonium salts on the photovoltaic performance for complete devices in a $\mathrm{FTO} / \mathrm{c}-\mathrm{TiO}_{2} / \mathrm{m}-\mathrm{TiO}_{2} /$ perovskite/spiro-OMeTAD/Au configuration (for more details see Methods). The data are shown in Fig. 5a in comparison to a control device without passivation (see Supplementary Table 1 for a comparison of all treatments with different concentrations).

The open-circuit voltages of all treated devices are significantly increased with an average improvement of $30 \mathrm{mV}, 70 \mathrm{mV}$, and 40 $\mathrm{mV}$, for EAI, IAI, and GuaI, respectively (see Fig. $5 \mathrm{~d}$ ). While the Gual-treated devices showed an average reduction of the FF by $2 \%$ absolute, the FF increased by 3.5 and $2 \%$ for EAI and IAItreated devices, respectively, reaching up to $81 \%$ on the best performing EAI device without significant loss of the short circuit current $\left(J_{\mathrm{sc}}\right)$. The short circuit photocurrent densities are barely modified with a maximum average decrease of $0.4 \mathrm{~mA} \mathrm{~cm}^{-2}$ for the IAI-treated devices. Overall, the mean efficiency increased from $20.5 \%$ for the control device to the $22.3 \%, 22.1 \%$, and $21.0 \%$ for EAI, IAI, and GuaI-treated devices, respectively, with the champion device treated via EAI achieving up to $22.3 \%$ PCE. Figure $5 \mathrm{~b}$ further confirms stable power output for all treated devices during maximum power point tracking under one sun illumination for one minute. The incident photon-to-current efficiency (IPCE) and integrated current density as a function of wavelength are shown in Fig. 5c. In agreement to UV-Vis, no shift in the onset of the IPCE spectra is detected for treated devices 

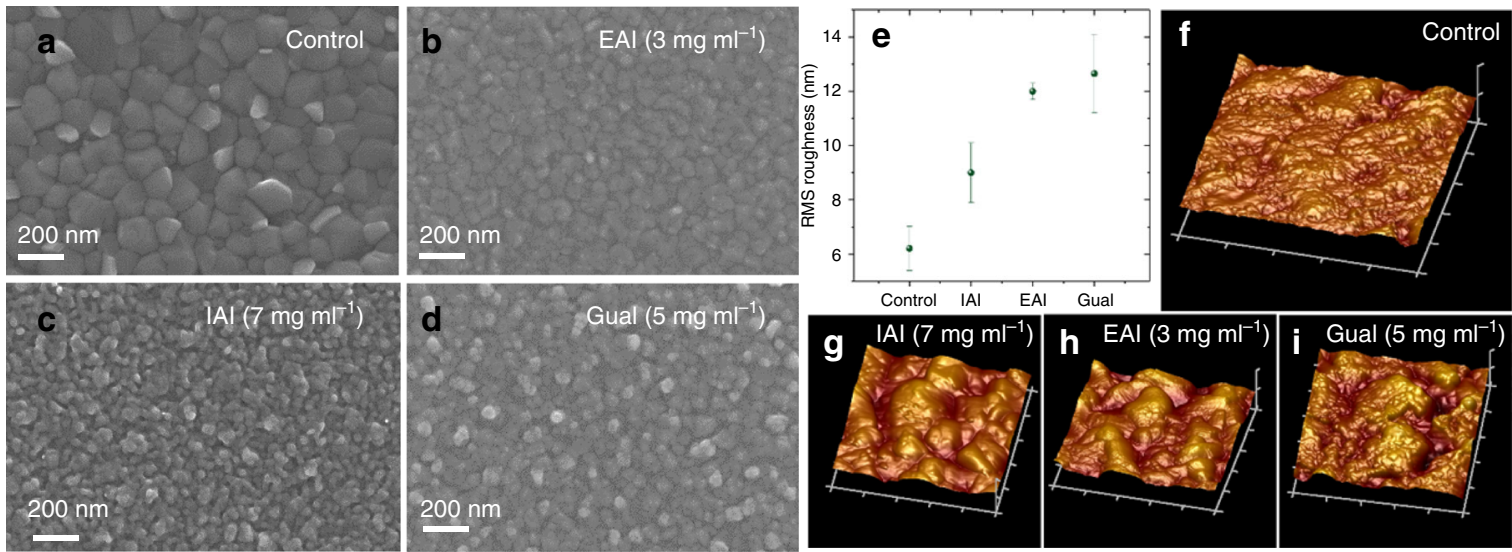

Fig. 3 Morphological characterization of perovskite layers: a-d SEM top view images of the perovskite films with optimized treatment conditions. e AFM measurements $(\mathbf{x}-\mathbf{y}: 1 \times 1 \mu \mathrm{m}, \mathrm{z}: 0 \pm 90 \mathrm{~nm})$ reveal increasing RMS surface roughness for surface-treated perovskite absorbers: $\mathbf{f}$ control, $\mathbf{g} \mid \mathrm{Al}, \mathbf{h} \mathrm{EAI}$, and i Gual
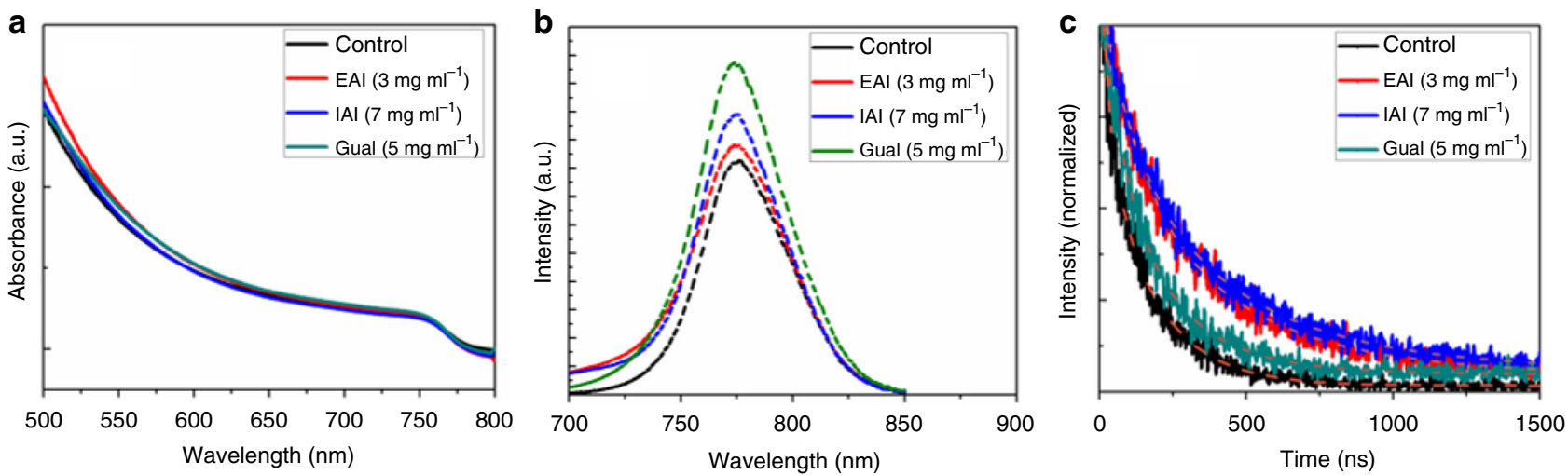

Fig. 4 Optical properties of perovskite films: a Absorbance, $\mathbf{b}$ steady-state photoluminescence, and $\mathbf{c}$ time-resolved photoluminescence spectra of treated and untreated perovskite films; dashed lines present the fitting curves

while integrated current densities agree well with the $J_{\text {sc }}$-values derived from the $J V$ measurements. Moreover, Supplementary Fig. 6a-d and Supplementary Table 2 show that the use of these ammonium salts as surface treatment is capable of reducing the hysteresis. To further analyze the effect of these buffer layers on the electrical properties, we performed intensity modulated voltage spectroscopy (IMVS). The data shown in Supplementary Fig. 6e agree with the trend found for $V_{o c}$, i.e., the devices showing higher $V_{\text {oc }}$ show a longer electron lifetime at $V_{\text {oc }}$.

Operational stability of perovskite solar cells. We also investigated is the operational stability of our PSCs under working conditions. This remains a major concern for PSC, which needs to be urgently addressed ${ }^{33}$. Best performing devices were subjected to full sunlight intensity with maximum power tracking for $550 \mathrm{~h}$ at room temperature in a nitrogen atmosphere.

We note the good stability of the control device preserving $80 \%$ of its initial PCE value. This is attributed to the perovskite composition employed in this work that has an extremely low content of MAI and contains a small addition of cesium iodide (CsI) to stabilize the predominately formamidinium lead iodide perovskite phase. From Fig. 6a, the passivated devices show a different behavior, a small initial performance drop being followed by a stable power output. Remarkably for the EAItreated device the overall loss in PCE is only $5 \%$. By contrast the control device shows a small but constant drop over the whole duration of the test. It is well-established that this behavior is due to the migration of ions from the HTM (LiTFSI) and gold from the gold electrode into the perovskite layer and to the $\mathrm{TiO}_{2}$ working electrode ${ }^{34}$. The fact that the efficiency loss for the passivated devices ceases after an initial decrease, it is reasonable to assume that that the passivating layer introduces a barrier at the HTM/perovskite interface that prevents the diffusion of HTM additives and gold diffusion into the perovskite layer. From crosssectional SEM images (Fig. 6b-e) we can see that before aging both control and modified devices appeared to have compact absorber layers. After $550 \mathrm{~h}$ or MPP testing, however, the appearance of voids at the interface between the mesoporous $\mathrm{TiO}_{2}$ and perovskite in the control and few voids also in the IAIand GuaI-treated perovskites is visible. These voids, stemming from the degradation of the perovskite absorber, may affect the photon absorption and act as recombination centers to hinder charge carrier collection during operation of the solar cell. Confirming this trend, EAI showed good stability at MPP under constant illumination with a decrease of only $5 \%$ and the absence of voids in the cross section SEM image of the aged devices shown in Fig. 6c.

\section{Discussion}

In summary, we investigated the effect of different ammonium salts (i.e., EAI, IAI, and GuaI) as surface passivation agents on mixed-cation and mixed-halide perovskite films. Solid-state NMR has evidenced that they form a tightly adhering lowdimensionality passivation layer of on the preexisting perovskite 

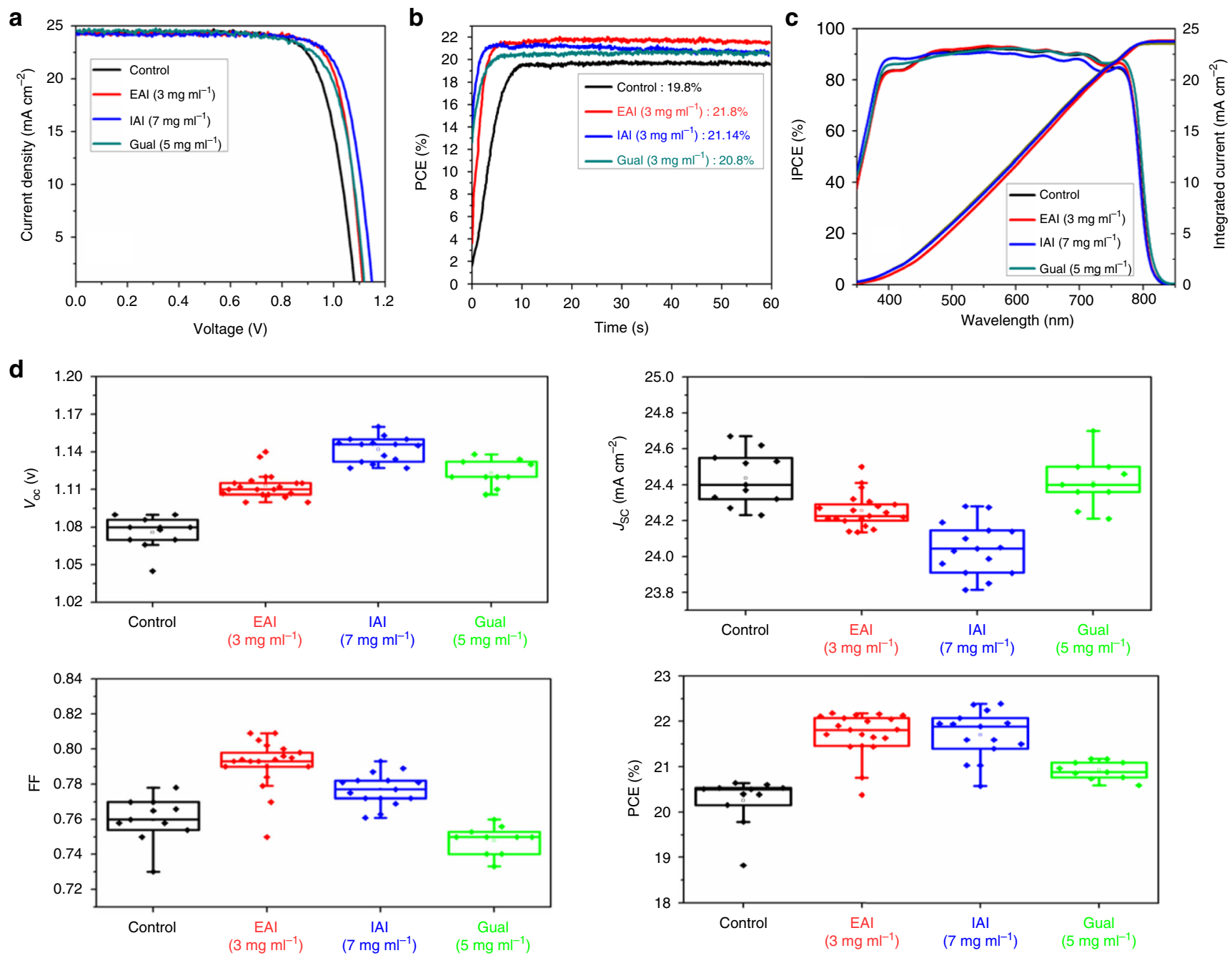

Fig. 5 Photovoltaic characterization: a J-V curves of the devices with different passivation layers in comparison to the control device without any treatment, with a scan rate of $10 \mathrm{mV} / \mathrm{s}$ b Maximum power tracking of the same device, $\mathbf{c}$ ) IPCE spectra and calculated photocurrent integrated over the standard AM $1.5 \mathrm{G}$ solar emission. In agreement to the $J-V$ data no change in the spectra is observed, and $\mathbf{d} J-V$ metrics of perovskite devices with different treatment in comparison to the control

grains. The introduction of these buffer layers showed a significant enhancement of the open-circuit voltage by $30 \mathrm{mV}$ (EAI), $70 \mathrm{mV}$ (IAI), and $40 \mathrm{mV}$ (GuaI), leading to the realization of PCEs as high as $22.3 \%, 22.1 \%$, and $21.0 \%$ for EAI, IAI, and GuaItreated devices, respectively. EAI and IAI showed an improvement also on the $\mathrm{FF}(+3.5 \%$ and $+2 \%$, respectively) with EAI reaching a $\mathrm{FF}$ of $81 \%$ on the best performing device. In contrast to the control sample, all passivated devices, after an initial drop, stabilized their efficiency with the EAI-treated device only losing $5 \%$ of its initial value after $550 \mathrm{~h}$ of MPP tracking. This work exemplifies the importance of interface engineering for perovskite solar cells and should stimulate other successful developments in the future.

\section{Methods}

Materials. All materials were purchased from Sigma-Aldrich and used as received, unless stated otherwise.

Solar cell preparation. Fluorine-doped tin oxide (FTO)-glass substrates (TCO glass, NSG 10, Nippon sheet glass, Japan) were cleaned by ultrasonication in Hellmanex (2\%, deionized water), rinsed thoroughly with deionized water and ethanol, and then treated in oxygen plasma for $15 \mathrm{~min}$. Thirty nanometer blocking layer $\left(\mathrm{TiO}_{2}\right)$ was sprayed on the cleaned FTO by at $450^{\circ} \mathrm{C}$ using a commercial titanium diisopropoxide bis(acetylacetonate) solution (75\% in 2-propanol, SigmaAldrich) diluted in anhydrous ethanol (1:9 volume ratio). A $150 \mathrm{~nm}$ mesoporous $\mathrm{TiO}_{2}$ layer (diluted paste (1:6 wt. ratio) (Dyesol 30NRD: ethanol)) spin coated at $5000 \mathrm{rpm}$ for $15 \mathrm{~s}$, and then sintered at $450^{\circ} \mathrm{C}$ for $30 \mathrm{~min}$ in dry air.

Synthesis of perovskite films. The perovskite films were deposited using a singlestep deposition method from the precursor solution, which was prepared in Argon atmosphere and containing $1.35 \mathrm{M}$ of FAI, FABr, MAI, CsI, $\mathrm{PbI}_{2}$ and $\mathrm{PbBr}_{2}$ in anhydrous dimethylformamide/ dimethylsulphoxide (4:1 (volume ratio)) to achieve the desired composition: $\left.\mathrm{FA}_{0.9} \mathrm{Cs}_{0.07} \mathrm{MA}_{0.03} \mathrm{~Pb} \mathrm{I}_{0.92} \mathrm{Br}_{0.08}\right)_{3}\left(3 \% \mathrm{PbI}_{2}\right.$ excess). The device fabrication, including the surface treatment step, was carried out inside a dry air box, under controlled atmospheric conditions with humidity $<2 \%$.Perovskite solution was spin-coated in a two-step program at 1000 and 6000 $\mathrm{rpm}$, respectively. Two hundred microliter of chlorobenzene was dropped on the spinning substrate. This was followed by annealing the films at $150^{\circ} \mathrm{C}$ for $30-40$ min. After preparing the initial perovskite layer (control) as described above, the film was cooled down at room temperature. Then, the surface treatment was performed by spin-coating a EAI, IAI, and GuaI-solution in isopropanol at different concentrations of $0 \mathrm{mg} \mathrm{ml}^{-1}, 3 \mathrm{mg} \mathrm{ml}^{-1}, 5 \mathrm{mg} \mathrm{ml}^{-1}, 7 \mathrm{mg} \mathrm{ml}^{-1}$, and $10 \mathrm{mg}$ $\mathrm{ml}^{-1}$ at $6000 \mathrm{rpm}$ for $30 \mathrm{~s}$, followed by annealing at $70^{\circ} \mathrm{C}$ for $10 \sim 15 \mathrm{~min}$. For completing the fabrication of devices, $85 \mathrm{mg}$ of $2,2^{\prime}, 7,7^{\prime}$-tetrakis( $N, N$-di- $p$-methoxyphenylamine)-9,9-spirobifluorene (spiro-OMeTAD) was dissolved in $1 \mathrm{ml}$ of chlorobenzene as a hole-transporting material (HTM). The HTM was spin coated at $4000 \mathrm{rpm}$ for $20 \mathrm{~s}$. The HTM was doped with bis(trifluoromethylsulfonyl)imide lithium salt ( $17.8 \mu \mathrm{l}$ prepared by dissolving $520 \mathrm{mg}$ LiTFSI in $1 \mathrm{ml}$ of acetonitrile), and $28.8 \mu \mathrm{l}$ of 4 -tert-butylpyridine. Finally, a $\sim 80 \mathrm{~nm}$ gold ( $\mathrm{Au}$ ) layer was thermally evaporated. 


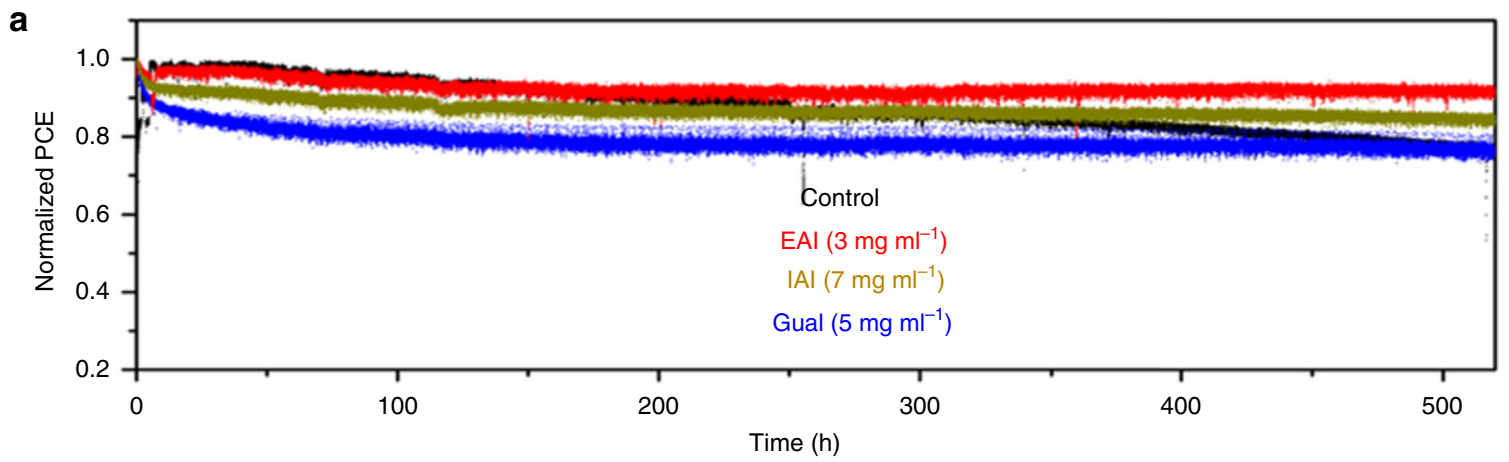

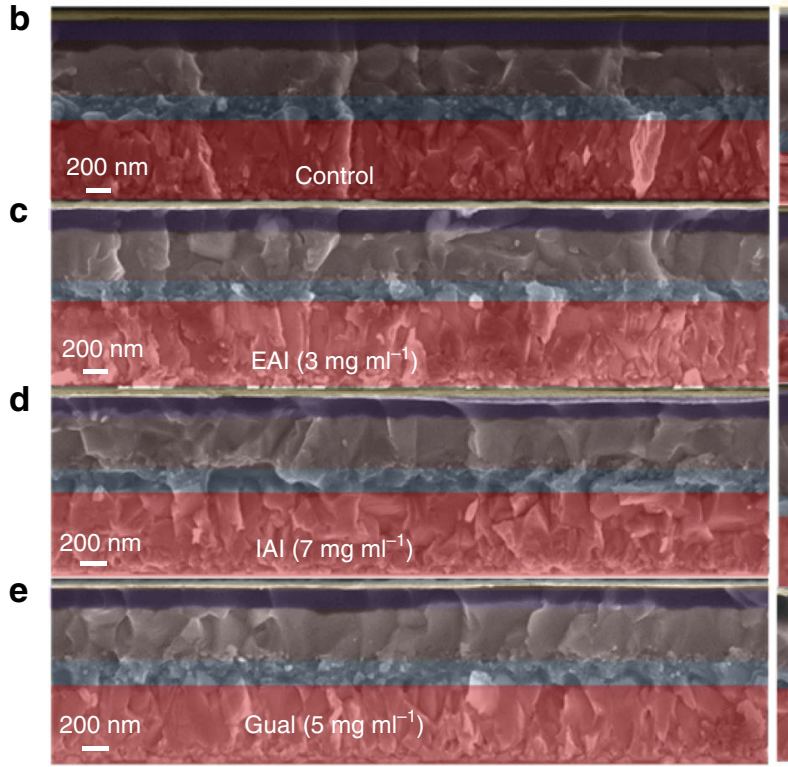

Before aging

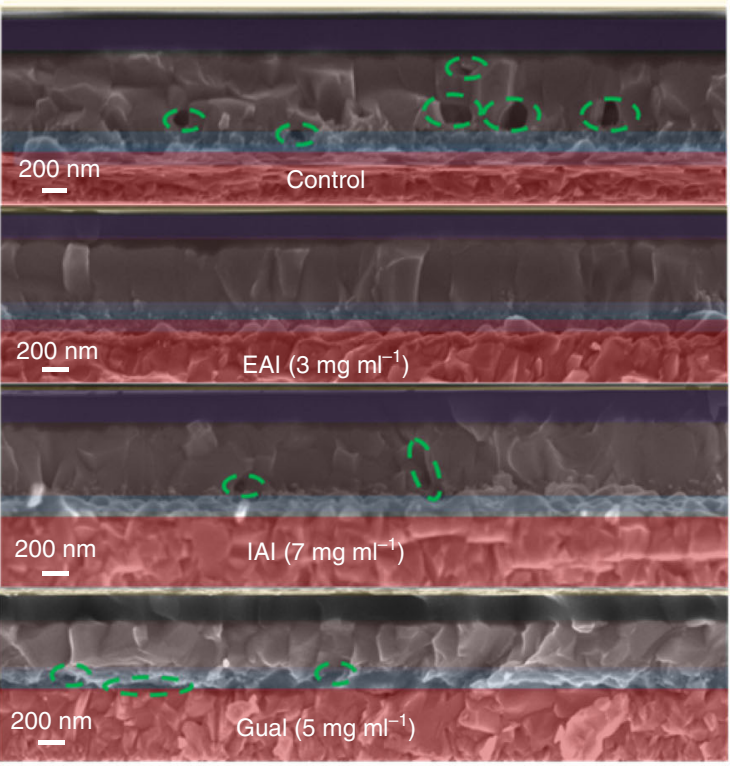

After aging

Fig. 6 Stability data and cross section before and after aging for control and treated devices: a A comparison of operational stability of control and treated perovskite devices. The devices were measured under a nitrogen environment at room temperature under constant illumination (LED source, 1 Sun) at a maximum power point for $550 \mathrm{~h}$. b, c, d, e Cross-sectional SEM images of the control and modified perovskite devices before and after aging at room temperature under constant illumination (LED source, 1 Sun) for $550 \mathrm{~h}$. Images show perovskite absorber and covering HTL

Device characterization. The current-voltage $(J-V)$ characteristics of the perovskite devices were recorded under ambient temperature and air conditions with a digital source meter (Keithley model 2400, USA). A $450 \mathrm{~W}$ xenon lamp (Oriel, USA) was used as the light source for photovoltaic $(J-V)$ measurements. The spectral output of the lamp was filtered using a Schott K113 Tempax sunlight filter (Präzisions Glas \& Optik GmbH, Germany) to reduce the mismatch between the simulated and actual solar spectrum to less than $2 \%$. The photo-active area of $0.16 \mathrm{~cm}^{2}$ was defined using a dark-colored metal mask.

Incident photon-to-current efficiency (IPCE). It was recorded under a constant white light bias of approximately $5 \mathrm{~mW} \mathrm{~cm}^{-2}$ supplied by an array of white light emitting diodes. The excitation beam coming from a $300 \mathrm{~W}$ Xenon lamp (ILC Technology) was focused through a Gemini- 180 double monochromator (Jobin Yvon Ltd) and chopped at $\sim 2 \mathrm{~Hz}$. The signal was recorded using a Model SR830 DSP Lock-In Amplifier (Stanford Research Systems).

Scanning electron microscopy (SEM). It was performed on a ZEISS Merlin HRSEM.

Atomic force microscopy (AFM). AFM images were obtained using a Bruker Dimension Icon Atomic Force Microscope in tapping mode.

X-ray powder diffractions were recorded on an X'Pert MPD PRO (Panalytical) equipped with a ceramic tube ( $\mathrm{Cu}$ anode, $\lambda=1.54060 \AA$ ), a secondary graphite (002) monochromator and a RTMS X'Celerator (Panalytical).

$\mathrm{UV}-\mathrm{Vis}$ measurements (Uv-Vis) were performed on a Varian Cary 5.
Photoluminescence spectra (PL) were obtained with a Florolog 322 (Horiba Jobin Ybon Ltd) in the wavelength range from 500 to $850 \mathrm{~nm}$ by exciting at $460 \mathrm{~nm}$.

Time-resolved photoluminescence (TRPL) was measured with a spectrometer (FluoroLog-3, Horiba) working in a time-correlated single-photon counting mode with less than ns time resolution. A picosecond pulsed diode laser head NanoLED N-670L (Horiba) emitting less than 200 ps duration pulses at $670 \mathrm{~nm}$ with a maximum repetition rate of $1 \mathrm{MHz}$ was used as excitation source. The measurements were carried out under ambient conditions and no change in PL was observed during the course of the measurements (several hours) indicating no appreciable decomposition.

Solid-state NMR measurements. Room temperature ${ }^{1} \mathrm{H}(900.0 \mathrm{MHz}) \mathrm{NMR}$ spectra were recorded on a Bruker Avance Neo 21.1 T spectrometer equipped with a $3.2 \mathrm{~mm}$ and $1.3 \mathrm{~mm}$ CPMAS probe. ${ }^{1} \mathrm{H}$ chemical shifts were referenced to solid adamantane $(\delta=1.91 \mathrm{ppm})$. Quantitative ${ }^{1} \mathrm{H}$ spectra were acquire with a recycle delay of $150 \mathrm{~s}$. The CsFA and CsMAFA samples were prepared as thin films on glass slides using the same deposition technique as for PSC fabrication and scratched off the slides into a rotor. $\mathrm{EAPbI}_{3}$ and $\mathrm{FA}_{0.9} \mathrm{EA}_{0.10} \mathrm{PbI}_{3}$ were prepared using mechanosynthesis, according to previously published procedures ${ }^{21,26,35-37}$. ${ }^{1} \mathrm{H}-{ }^{1} \mathrm{H}$ spin diffusion measurements at $20 \mathrm{kHz}$ MAS were carried out using a mixing period of $50 \mathrm{~ms}$ and a recycle delay of $1 \mathrm{~s}\left(\mathrm{FA}_{0.90} \mathrm{EA}_{0.10} \mathrm{PbI}_{3}\right) .{ }^{1} \mathrm{H}-{ }^{1} \mathrm{H}$ spin diffusion measurements at $60 \mathrm{kHz}$ MAS used a mixing period of $4 \mathrm{~s}$ and a recycle delay of $3 \mathrm{~s}$ (CsFA(I) thin film).

IMVS measurements were performed by Bio-Logic SP300 in combination with the Galvano Staircase Spectroscopy routine from EC-Lab Software. Further description of the technique is provided in Supplementary Note 2. 
Long-term light soaking test. Stability measurements were performed with a Biologic MPG2 potentiostat under a full AM 1.5 Sun-equivalent white LED lamp. The devices were measured with a maximum power point (MPP) tracking routine under continuous illumination at room temperature. The MPP was updated every $10 \mathrm{~s}$ by a standard perturb and observe method. Every minute a $J V$ curve was recorded in order to track the evolution of individual $J V$ parameters.

\section{Data availability}

Data that support the findings of this study are available in separate Supplementary Data Files in Supplementary Information section. All other relevant data are available from the corresponding authors upon reasonable request.

Received: 24 November 2018 Accepted: 28 May 2019

Published online: 08 July 2019

\section{References}

1. Burschka, J. et al. Sequential deposition as a route to high-performance perovskite-sensitized solar cells. Nature 499, 316-319 (2013).

2. Saliba, M. et al. Cesium-containing triple cation perovskite solar cells: improved stability, reproducibility and high efficiency. Energy Environ. Sci. 9 , 1989-1997 (2016).

3. Park, N.-G., Gratzel, M., Miyasaka, T., Zhu, K. \& Emery, K. Towards stable and commercially available perovskite solar cells. Nat. Energy 1, 16152 (2016).

4. Tsai, H. et al. High-efficiency two-dimensional Ruddlesden- Popper perovskite solar cells. Nature 536, 312-316 (2016).

5. Bi, D. et al. Efficient luminescent solar cells based on tailored mixed-cation perovskites. Sci. Adv. 2, e1501170-e1501170 (2016)

6. chart, NREL. http://www.nrel.gov/ncpv/images/efficiency_chart.jpg. (2019).

7. Zheng, X. et al. Defect passivation in hybrid perovskite solar cells using quaternary ammonium halide anions and cations. Nat. Energy 2, 17102-17109 (2017).

8. Dar, M. I. et al. Function follows form: correlation between the growth and local emission of perovskite structures and the performance of solar cells. $A d v$. Funct. Mater. 27, 1701433-1701439 (2017).

9. Dualeh, A. et al. Impedance spectroscopic analysis of lead iodide perovskitesensitized solid-state solar cells. ACS Nano 8, 362-373 (2013).

10. Arora, N. et al. Intrinsic and extrinsic stability of formamidinium lead bromide perovskite solar cells yielding high photovoltage. Nano Lett. 16 , 7155-7162 (2016).

11. Chen, J., Seo, J.-Y. \& Park, N.-G. Simultaneous improvement of photovoltaic performance and stability by in situ formation of 2D perovskite at (FAPbI 3 ) $0.88(\mathrm{CsPbBr} 3)$ 0.12/CuSCN. Interface Adv. Energy Mater. 8, 1702714-1702715 (2018).

12. R. J., S. et al. Enhancing defect tolerance and phase stability of highbandgap perovskites via guanidinium alloying. ACS Energy Lett. 3, 1261-1268 (2018).

13. Cho, K. T. et al. Highly efficient perovskite solar cells with a compositionally engineered perovskite/hole transporting material interface. Energy Environ. Sci. 10, 621-627 (2017).

14. Hawash, Z. et al. Interfacial modification of perovskite solar cells using an ultrathin MAI layer leads to enhanced energy level alignment, efficiencies, and reproducibility. J. Phys. Chem. Lett. 8, 3947-3953 (2017).

15. Wang, F. et al. Phenylalkylamine passivation of organolead halide perovskites enabling high-efficiency and air-stable photovoltaic cells. Adv. Mater. 28, 9986-9992 (2016).

16. Han, G. et al. Additive selection strategy for high performance perovskite photovoltaics. J. Phys. Chem. C. 122, 13884-13893 (2018).

17. Wang, Q., Dong, Q., Li, T., Gruverman, A. \& Huang, J. Thin insulating tunneling contacts for efficient and water-resistant perovskite solar cells. Adv. Mater. 28, 6734-6739 (2016).

18. De Marco, N. et al. Guanidinium: a route to enhanced carrier lifetime and open-circuit voltage in hybrid perovskite solar cells. Nano Lett. 16, 1009-1016 (2016).

19. Hou, X. et al. Effect of guanidinium on mesoscopic perovskite solar cells. J. Mater. Chem. A 5, 73-78 (2017)

20. Jodlowski, A. D. et al. Large guanidinium cation mixed with methylammonium in lead iodide perovskites for $19 \%$ efficient solar cells. Nat. Energy 2, 972-979 (2017).

21. Kubicki, D. J. et al. Formation of Stable mixed guanidinium-methylammonium phases with exceptionally long carrier lifetimes for high-efficiency lead iodide-based perovskite photovoltaics. J. Am. Chem. Soc. 140, 3345-3351 (2018).
22. Hsu, H.-L. et al. High-performance and high-durability perovskite photovoltaic devices prepared using ethylammonium iodide as an additive. J. Mater. Chem. A 3, 9271-9277 (2015).

23. Poorkazem, K. \& Kelly, T. L. Compositional engineering to improve the stability of lead halide perovskites: a comparative study of cationic and anionic dopants. ACS Appl. Energy Mater. 1, 181-190 (2017).

24. Wang, Q. et al. Enhancing efficiency of perovskite solar cells by reducing defects through imidazolium cation incorporation. Mater. Today Energy 7 , 161-168 (2018)

25. Zhang, Y. et al. Auto-passivation of crystal defects in hybrid imidazolium/ methylammonium lead iodide films by fumigation with methylamine affords high efficiency perovskite solar cells. Nano Energy 58, 105-111 (2019).

26. Kubicki, D. J. et al. Cation dynamics in mixed-cation (MA) x(FA) 1- xPbI 3 Hybrid perovskites from solid-state NMR. J. Am. Chem. Soc. 139, 10055-10061 (2017)

27. Tavakoli, M. M. et al. Adamantanes enhance the photovoltaic performance and operational stability of perovskite solar cells by effective mitigation of interfacial defect states. Adv. Energy Mater. 8, 1800275-1800277 (2018).

28. Bi, D. et al. Multifunctional molecular modulators for perovskite solar cells with over $20 \%$ efficiency and high operational stability. Nat. Commun. 9, 4482 (2018)

29. Im, Jeong-Hyeok, Chung, Jaehoon, Kim, Seung-Joo \& Park, Nam-Gyu Synthesis, structure, and photovoltaic property of a nanocrystalline $2 \mathrm{H}$ perovskite-type novel sensitizer $\left(\mathrm{CH}_{3} \mathrm{CH}_{2} \mathrm{NH}_{3}\right) \mathrm{PbI}_{3}$. Nanoscale Res. Lett. 7 353 (2012).

30. Fan, J. et al. Thermodynamically self-healing 1D-3D hybrid perovskite solar cells. Adv. Energy Mater. 8, 1703421-1703428 (2018).

31. Chen, Y. et al. 2D Ruddlesden-Popper perovskites for optoelectronics. $A d v$. Mater. 30, 1703487-15 (2017).

32. Elena, B., Pintacuda, G., Mifsud, N. \& Emsley, L. Molecular structure determination in powders by NMR crystallography from proton spin diffusion. J. Am. Chem. Soc. 128, 9555-9560 (2006).

33. Domanski, K., Alharbi, E. A., Hagfeldt, A., Gratzel, M. \& Tress, W. Systematic investigation of the impact of operation conditions on the degradation behaviour of perovskite solar cells. Nat. Energy 3, 61-67 (2018).

34. Domanski, K. et al. Not all that glitters is gold: metal-migrationinduced degradation in perovskite solar cells. ACS Nano 10, 6306-6314 (2016).

35. Prochowicz, D. et al. Mechanosynthesis of the hybrid perovskite $\mathrm{CH} 3 \mathrm{NH}$ $3 \mathrm{PbI}$ 3: characterization and the corresponding solar cell efficiency. J. Mater. Chem. A 3, 20772-20777 (2015).

36. Kubicki, D. J. et al. Phase segregation in Cs-, Rb- and K-doped mixed-cation (MA) x(FA) 1-xPbI 3hybrid perovskites from solid-state NMR. J. Am. Chem. Soc. 139, 14173-14180 (2017).

37. Kubicki, D. J. et al. Phase segregation in potassium-doped lead halide perovskites from 39K solid-state NMR at 21.1 T. J. Am. Chem. Soc. 140 $7232-7238$ (2018)

\section{Acknowledgements}

E.A. and A.Q.A gratefully acknowledge King Abdulaziz City for Science and Technology (KACST) for a fellowship. M.G. and S.M.Z thank the King Abdulaziz City for Science and Technology (KACST) for the financial support. A.R.U. acknowledges the financial support from the Swiss National Science Foundation (SNSF) under project number P3P3P2_177790. D.J.K., B.J.W., and L.E. acknowledge Swiss National Science Foundation Grants No. 200021_160112 \& 200020_178860 and the European Union's Horizon 2020 research and innovation programme under grant agreement No 764047. E.A. and F.G. acknowledge Dr. Daniel Prochowicz and Anand Agarwalla for help with XRD and stability measurements, respectively. Authors appreciate the support of Guido Rothenberger (EPFL) on devising the model for the kinetic analysis of time-resolved photoluminescence decay.

\section{Author contributions}

E.A. and M.G. conceived the idea. E.A. optimized the composition and designed the experiments. J.L. took the SEM images. F.G. conducted the IMVS measurements. A.Q.A and M.H.A. contributed in the device fabrications. A.Y.A., A.A., and H.A. prepared the films and the recorded the AFM measurements. A.R.U. assessed sample roughness and analyzed AFM results. A.B. conducted TRPL measurements. D.J.K, B.J.W., and L.E. carried out and analysed the solid-state NMR measurements. J-E.M. supervised A.B. E.A. wrote the first manuscript. M.G., S.M.Z., F.G., and A.Y.A. supervised the project and results discussion. All the authors contributed to the preparation of the manuscript. 


\section{Additional information}

Supplementary Information accompanies this paper at https://doi.org/10.1038/s41467019-10985-5.

Competing interests: The authors declare no competing interests.

Reprints and permission information is available online at http://npg.nature.com/ reprintsandpermissions/

Peer review information: Nature Communications thanks Jong Hyeok Park and other anonymous reviewer(s) for their contribution to the peer review of this work.

Publisher's note: Springer Nature remains neutral with regard to jurisdictional claims in published maps and institutional affiliations. (c) (i) Open Access This article is licensed under a Creative Commons Attribution 4.0 International License, which permits use, sharing, adaptation, distribution and reproduction in any medium or format, as long as you give appropriate credit to the original author(s) and the source, provide a link to the Creative Commons license, and indicate if changes were made. The images or other third party material in this article are included in the article's Creative Commons license, unless indicated otherwise in a credit line to the material. If material is not included in the article's Creative Commons license and your intended use is not permitted by statutory regulation or exceeds the permitted use, you will need to obtain permission directly from the copyright holder. To view a copy of this license, visit http://creativecommons.org/ licenses/by/4.0/.

(C) The Author(s) 2019 